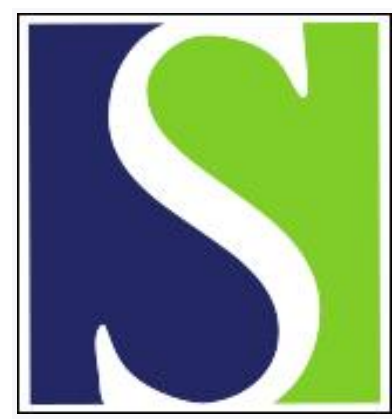

Scand J Work Environ Health 2013;39(1):88-95

https://doi.org/10.5271/sjweh.3304

Published online: 08 May 2012, Issue date: Jan 2013

Occupational lifting and pelvic pain during pregnancy: a study within the Danish National Birth Cohort

by Larsen PS, Strandberg-Larsen K, Juhl M, Svendsen SW, Bonde JP, Nybo Andersen A-M

Affiliation: Section of Social Medicine, Department of Public Health, University of Copenhagen, Øster Farimagsgade 5, DK-1014 Copenhagen K, Denmark. pernillelarsen@sund.ku.dk

Refers to the following text of the Journal: 1989;15(6):404-414

The following article refers to this text: 2014;40(1):96-99

Key terms: Danish National Birth Cohort; lifting; occupation; occupational lifting; parity; pelvic pain; physical activity; physical workload; pregnancy; pregnant women

This article in PubMed: www.ncbi.nlm.nih.gov/pubmed/22569778

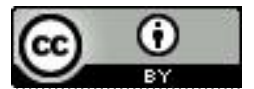




\title{
Occupational lifting and pelvic pain during pregnancy: a study within the Danish National Birth Cohort
}

\author{
by Pernille Stemann Larsen, MSc, ${ }^{1}$ Katrine Strandberg-Larsen, MSc, PhD, ${ }^{1}$ Mette Juhl, MPH, PhD, ${ }^{1}$ \\ Susanne Wulff Svendsen, MD, PhD, ${ }^{2}$ Jens Peter Bonde, MD, PhD, ${ }^{3}$ Anne-Marie Nybo Andersen MD, PhD ${ }^{1}$
}

\begin{abstract}
Larsen PS, Strandberg-Larsen K, Juhl M, Svendsen SW, Bonde JP, Nybo Andersen A-M. Occupational lifting and pelvic pain during pregnancy: a study within the Danish National Birth Cohort. Scand J Work Environ Health. 2013;39(1):88-95. doi:10.5271/sjweh.3304
\end{abstract}

Objective Pelvic pain during pregnancy is a common ailment, and the disease is a major cause of sickness absence during pregnancy. It is plausible that occupational lifting may be a risk factor of pelvic pain during pregnancy, but no previous studies have examined this specific exposure. The aim of this study was to examine the association between occupational lifting and pelvic pain during pregnancy.

Methods The study comprised 50143 pregnant women, enrolled in the Danish National Birth Cohort in the period from 1996-2002. During pregnancy, the women provided information on occupational lifting (weight load and daily frequency), and six months post partum on pelvic pain. Adjusted odds ratios for pelvic pain during pregnancy according to occupational lifting were calculated by logistic regression.

Results Any self-reported occupational lifting ( $>1$ time/day and loads weighing $>10 \mathrm{~kg}$ ) was associated with an increased risk of pelvic pain during pregnancy as compared to no such lifting. A confounder-adjusted exposure-response relation was observed between self-reported total loads lifted and pelvic pain during pregnancy. Daily lifting of both medium (11-20 kg) and heavy loads $(>20 \mathrm{~kg})$ were associated with increased risk, and the highest risk was observed among women who lifted heavy loads independent of exposure to medium loads.

Conclusion Occupational lifting may increase the risk of pelvic pain during pregnancy.

Key terms occupation; parity; physical activity; physical workload; pregnant women.

During pregnancy, physical workload may be a risk factor for several adverse pregnancy outcomes, such as spontaneous abortion, stillbirth, pre-term birth, and low birth weight (1). Physical workload may, however, also have a negative impact on maternal health (2-4). About $20 \%$ of all pregnant women suffer from pelvic pain, and in Scandinavia the disease is a major cause of sickness absence during pregnancy (5-7). For many women, the disease interferes with the ability to carry out daily activities, and in some cases this progresses to chronic post-partum pain (7-9). The etiology is not well established, and there is a lack of international consensus about the nature of the disease and its identification and classification $(7,10)$. Over the last decades, the impact of sickness absence on quality of life and the subsequent socioeconomic costs has increased the attention of the medical community on this subject (11).

A few studies have examined the effects of physical workload on the risk of pelvic pain during pregnancy. These studies found that uncomfortable working conditions and physical strenuous workload were associated with increased risk $(2,12,13)$. During pregnancy, the extensive biomechanical changes lead to pelvic strain (14), and it is plausible that occupational lifting during pregnancy may increase this strain further. However, no studies have evaluated the effects of occupational lifting specifically. Regular leisure-time physical activity prior to pregnancy is associated with a decreased risk of pelvic pain (15), but during pregnancy it may have the opposite effect.

1 Section of Social Medicine, Department of Public Health, University of Copenhagen, Denmark.

2 Danish Ramazzini Centre, Department of Occupational Medicine, Herning Hospital, Denmark.

3 Department of Occupational and Environmental Medicine, Bispebjerg Hospital, Denmark.

Correspondence to: Pernille Stemann Larsen, Section of Social Medicine, Department of Public Health, University of Copenhagen, Øster Farimagsgade 5, DK-1014 Copenhagen K, Denmark. [E-mail: pernillelarsen@sund.ku.dk] 
The aim of this study was to (i) examine the association between occupational lifting and pelvic pain during pregnancy and (ii) study the possible modifying effects of leisure-time physical activity and parity, respectively.

\section{Methods}

\section{Study population}

This study was based on data from the Danish National Birth Cohort (DNBC). In the period from 1996-2002, 91386 women, representing 100418 pregnancies, were enrolled in the cohort. At the first antenatal care visit, the general practitioners briefed participants about the study and provided an informed consent form. By signing the informed consent form, the pregnant women agreed to be interviewed by telephone. The first pregnancy interview took place around the $12-16^{\text {th }}$ week of gestation, and - post partum - the women were interviewed when the child was approximately six months. Further information about the DNBC is available at the study website (www.dnbc.dk) (16).

Of the 100418 enrolled pregnancies, we restricted the analysis to women with data from both the pregnancy and 6-month interview ( $\mathrm{N}=67567)$. We excluded pregnancies of women (i) with no employment [defined as not working at all or working $<15$ hours per week $(\mathrm{N}=13$ 163)]; (ii) with incomplete information on their employment situation $(\mathrm{N}=27)$; (iii) who had been out of work for $>3$ months prior to the pregnancy interview due to sick absence, absence due to the nature of the work, parental leave, or education $(\mathrm{N}=1407)$; (iv) with incomplete information on time since being in work $(\mathrm{N}=18)$; and $(\mathrm{v})$ with incomplete information on job titles $(\mathrm{N}=557)$, any occupational lifting $(\mathrm{N}=117)$, total load lifted $(\mathrm{N}=968)$, pelvic pain during pregnancy $(\mathrm{N}=107)$, parity $(\mathrm{N}=24)$, occupational status $(\mathrm{N}=6)$, pre-pregnancy body mass index (BMI) $(\mathrm{N}=757)$, smoking during pregnancy $(\mathrm{N}=127)$, leisure-time heavy lifting $(\mathrm{N}=37)$, leisure-time physical activity $(\mathrm{N}=101)$, or working hours $(\mathrm{N}=8)$. Consequently, a total of 47935 women, representing 50143 pregnancies were included in this study.

\section{Main exposure}

In the pregnancy interview, the pregnant women were asked: "In your job, do you daily lift $>20$ kilos at a time?" If they responded yes, they were asked: "How many times a day, do you lift $>20$ kilos?" These questions were also asked for loads of $11-20 \mathrm{~kg}$.

We constructed four measures of occupational lifting: (i) Any self-reported occupational lifting $>1$ time/day and weighing $>10 \mathrm{~kg}$ (no/yes); (ii) Self-reported total load lifted, where we combined information on medium and heavy loads lifted with the frequency of daily lifts $(0,15-100,101-200,201-500,501-1000,>1000 \mathrm{~kg} /$ day). When we calculated the self-reported total load lifted, medium loads (11-20 kg) were set to $15 \mathrm{~kg}$, heavy loads ( $>20 \mathrm{~kg}$ ) were set to $22.5 \mathrm{~kg}$, and lifting $<15 \mathrm{~kg}$ or $<1$ time/day were regarded as "no lifting"; (iii) Selfreported frequency of lifting medium and heavy loads. This 9-category variable combined the frequency of lifting medium and heavy loads $(0,1-10,>10$ times/day); (iv) Total load lifted derived from a job exposure matrix (JEM) that cross-tabulated occupational titles from the Danish version of the International Standard Classification of Occupations (DISCO-88) with estimates of daily total load lifted according to the judgment of five experts (17). Since more than one job title could share the same 4-digit DISCO-88 code, we calculated the mean of the total load lifted for job titles with the same DISCO-88 code. The variable was categorized in the same way as the self-reported total load lifted.

\section{Outcome of interest}

In the 6-month interview, the women were asked the following screening question: "During your pregnancy or shortly after birth, did you suffer from pelvic pain that was so strong it affected your ability to walk?" Women who reported a positive answer to this screening question were regarded as having pelvic pain during pregnancy.

\section{Covariates}

We decided a priori to adjust the analyses for the following potential confounders: maternal age at conception $(<25,25-34,35-39, \geq 40$ years), parity $(0, \geq 1)$, occupational status (high-grade professionals, low-grade professionals, skilled workers, unskilled workers, students $)$, pre-pregnancy BMI $(<18.5,18.5-<25,25-<30$, $\left.>30 \mathrm{~kg} / \mathrm{m}^{2}\right)$, smoking during pregnancy $(0,<10, \geq 10$ cigarettes/day), physical activity during pregnancy $(0$, 1-60, >60 minutes/week), leisure-time heavy lifting ( $>20 \mathrm{~kg} /$ day), and working hours (day, evening, night, rotating shift work without night, rotating shift work with night). Furthermore, for the sub-analyses, we constructed a variable indicating any previous muscle joint disease (no/yes), and a variable indicating timing of the pregnancy interview $\left(1^{\text {st }}, 2^{\text {nd }}, 3^{\text {rd }}\right.$ trimester $)$.

\section{Statistical analysis}

We used logistic regression models to analyze the association between occupational lifting and pelvic pain during pregnancy. The analyses were conducted separately for each of the four exposure variables. We examined 
if any exposure-response relation could be observed by supplementing with tests for trend using exposure category as a continuous variable. An interaction analysis for the daily frequency of medium and heavy loads lifted was performed by including an interaction parameter in the logistic regression model. In addition, we examined the joint effects of being exposed to both medium and heavy loads by constructing a combined variable of the two exposures.

We examined any modifying effects of leisure-time physical activity and parity, respectively. We examined the joint effects of occupational lifting and leisure-time physical activity by constructing a combined variable of occupational lifting and leisure-time physical activity. In order to examine the consequences of non-independent observations, we performed a sub-analysis restricted to first time participation in the DNBC. In another subanalysis, we restricted the population to women with no previous muscle or joint disease in order to address reverse causation. Due to logistic circumstances, the women completed the pregnancy interview at different gestational ages - some women quite late during pregnancy. Therefore, we stratified the analyses according to the timing of this interview.

\section{Results}

Of the 50143 enrolled pregnant women, 17.8\% $(\mathrm{N}=8921)$ reported pelvic pain during pregnancy. These women were more often parous, lower-grade professionals, overweight, and exposed to leisure-time heavy lifting compared to women without pelvic pain during pregnancy (data not shown). Overall, according to self-report, 12970 (25.9\%) women were exposed to occupational lifting. Of these, $351(0.7 \%)$ women reported lifting a total load of $>1000 \mathrm{~kg}$ per day. Compared to non-lifters, women who reported exposure to any occupational lifting were more often of young age, parous, unskilled workers, overweight, smokers, physically inactive during leisure time, and more likely to be exposed to leisure-time heavy lifting (table 1).

Any self-reported occupational lifting was associated with an increased risk of pelvic pain during pregnancy [odds ratio (OR) 1.18, 95\% confidence interval (95\% CI) 1.12-1.25]. For self-reported total load lifted per day, an exposure-response relation was observed (P-value for trend $<0.0001$ ). Women, who reported a total load between 201-500 and 501-100 kg per day had the highest risk (1.45, 95\% CI 1.31-1.60, and OR 1.45, $95 \%$ CI $1.23-1.72$, respectively). For daily total loads lifted derived from the JEM, no association with pelvic pain during pregnancy was observed (table 2).

Compared to non-lifters the risk of pelvic pain dur- ing pregnancy increased as the frequency of daily lifts of medium and heavy loads lifted increased, respectively. Among women, who reported lifts of heavy loads $>10$ times/day, lifting seemed harmful independent of the daily frequency of medium loads lifted. In the remaining groups, the risk increased as the frequency of lifts and the level of loads increased. However, no statistically significant interaction was observed between the frequency of medium and heavy loads lifted (table 3).

An interaction between occupational lifting and leisure-time physical activity was observed. Among non-lifters, the risk decreased with an increasing level of physical activity (table 4). No signs of interaction were observed between occupational lifting and parity (data not shown). When we stratified the analyses by timing of the pregnancy interview, the risk increased with later time of interview, but the test of interaction did not reach statistical significance (table 5). Restriction to first-time participants in the DNBC and women without any previous muscles or joint disease, respectively, did not affect the results (data not shown).

\section{Discussion}

In this study based on data of around 50000 pregnancies, women who reported any occupational lifting had an increased risk of pelvic pain during pregnancy. The risk increased in an exposure-response manner with increasing level of total loads lifted per day.

No previous studies have focused on the relation between occupational lifting and the risk of pelvic pain during pregnancy specifically, but a few studies have examined the effects of physical workload. Associations between uncomfortable or physically strenuous work and pelvic pain during pregnancy were found in these studies, but no or only a modest effect was observed for occupational lifting $(2,12,13)$.

The inconsistency between the previous and present findings could be attributable to insufficient statistical power in previous studies, as these were smaller than the present study. However, differences in definitions of self-reported physical workload, and different disease definitions, varying from self-reported pain to diagnoses based on clinical examinations, may also contribute to the inconsistent results. Likewise, differences in confounder control may partly explain the inconsistency. Based on a review of the literature, we decided a priori to adjust for age at conception, parity, occupational status, pre-pregnancy BMI, smoking during pregnancy, leisure-time physical activity, leisure-time heavy lifting, and working hours. Additionally, we performed a Chi-squared test for independence $(\mathrm{P}<0.05)$ in order to verify the inclusion of the above covariates. The covari- 
Table 1. Distribution (\%) of selected characteristics according to self-reported pelvic pain and total load lifted (kg) per day during pregnancy. The Danish National Birth Cohort 1996-2002.

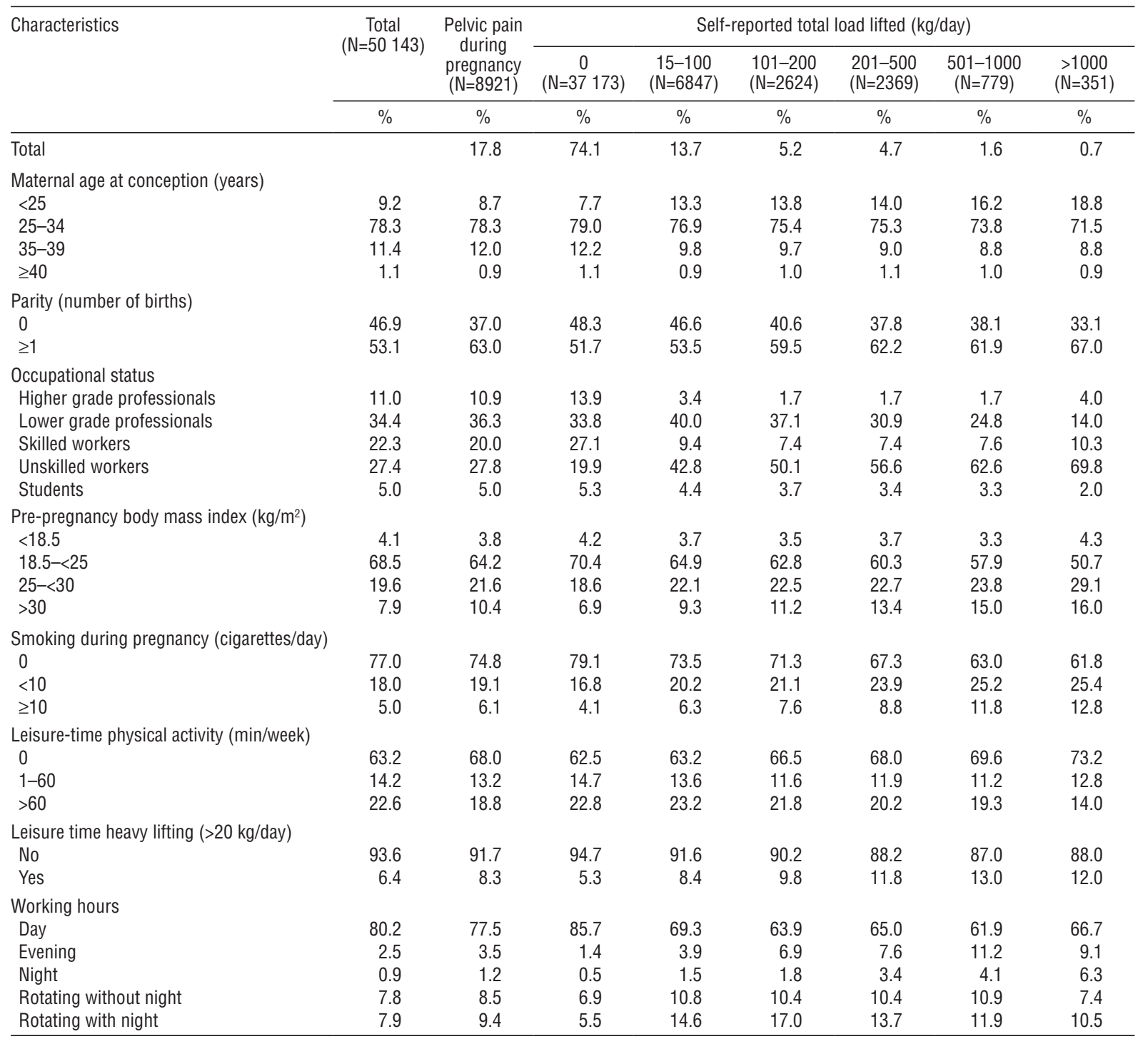

ates that confounded the estimate the most were: parity, overweight, smoking, and not working during day time, while the remaining factors had only a minor influence. In the literature, factors such as working in draft and cold, pushing and pulling, bending and twisting, stress/ depression, and family history of pelvic pain during pregnancy were also identified as risk factors of pelvic pain during pregnancy, but we had no information on these potential confounders and could not include them in the model $(2,12,18-20)$.

In the DNBC, women were asked to report exposure to physically strenuous work and standing/walking working postures. We decided, however, not to include these variables in the model because of the strong correlation between occupational lifting and both of these variables (one cannot perform lifting without perceiving oneself to be exposed to physical strenuous work and lifting in a sitting posture is unlikely). Statistically, the implication of such collinearity makes it very difficult to separate the effect of occupational lifting and these two variables (21). Furthermore, we did not adjust for previous pelvic pain, as this may partly be caused by occupational lifting and at the same time be correlated with pelvic pain in the present pregnancy (22).

The strengths of this study were the size of the population and the prospective study design. In this study, we did not collect information on the onset of pelvic pain in the first pregnancy interview because only a minority of women experience pelvic pain in the first trimester, and - as stated above - the first pregnancy interview was 
Table 2. Crude and adjusted odds ratios $(\mathrm{OR})$ for pelvic pain during pregnancy according to occupational lifting. The Danish National Birth Cohort, 1996-2002. [95\% Cl=95\% confidence interval]

\begin{tabular}{|c|c|c|c|c|c|}
\hline & \multicolumn{2}{|c|}{ Crude } & \multicolumn{2}{|c|}{ Adjusted } & \multirow[t]{2}{*}{$\mathrm{P}$-value } \\
\hline & OR & $95 \% \mathrm{Cl}$ & $\mathrm{OR}^{\mathrm{a}}$ & $95 \% \mathrm{Cl}$ & \\
\hline \multicolumn{6}{|c|}{ Any self-reported lifting } \\
\hline No & 1.00 & reference & 1.00 & reference & \\
\hline Yes & 1.31 & $1.24-1.37$ & 1.18 & $1.12-1.25$ & \\
\hline \multicolumn{6}{|c|}{ Self-reported total load lifted (kg/day) } \\
\hline 0 & 1.00 & reference & 1.00 & reference & $<0.0001$ \\
\hline $15-100$ & 1.13 & $1.05-1.21$ & 1.06 & $0.99-1.13$ & \\
\hline $101-200$ & 1.36 & $1.23-1.49$ & 1.21 & $1.09-1.34$ & \\
\hline $201-500$ & 1.65 & $1.50-1.82$ & 1.45 & $1.31-1.60$ & \\
\hline $501-1000$ & 1.70 & $1.44-2.00$ & 1.45 & $1.23-1.72$ & \\
\hline$>1000$ & 1.56 & $1.22-2.00$ & 1.31 & $1.02-1.69$ & \\
\hline \multicolumn{6}{|c|}{ Total load lifted (kg/day) derived from a job exposure matrix (JEM) } \\
\hline 0 & 1.00 & reference & 1.00 & reference & 0.0918 \\
\hline $15-100$ & 1.07 & $0.92-1.24$ & 1.02 & $0.88-1.20$ & \\
\hline $101-200$ & 0.88 & $0.70-1.10$ & 1.00 & $0.80-1.27$ & \\
\hline $201-500$ & 1.24 & $1.17-1.32$ & 1.10 & $1.03-1.18$ & \\
\hline $501-1000$ & 0.96 & $0.87-1.06$ & 0.97 & $0.87-1.07$ & \\
\hline$>1000$ & 1.12 & $1.04-1.20$ & 1.00 & $0.92-1.09$ & \\
\hline
\end{tabular}

${ }^{a}$ Adjusted for maternal age at conception, parity, occupational status, pre-pregnancy body mass index, smoking during pregnancy, leisure-time physical activity during pregnancy, leisure time heavy lifting, and working hours.

Table 3. Joint effects of frequency of self-reported medium $(11-20 \mathrm{~kg})$ and heavy $(>20 \mathrm{~kg})$ occupational loads lifted per day on risk of pelvic pain during pregnancy. The Danish National Birth Cohort, 1996-2002. [OR=0dds ratio: 95\% Cl=95\% confidence interval]

\begin{tabular}{|c|c|c|c|c|c|c|}
\hline \multirow{3}{*}{$\begin{array}{l}\text { Frequency of self-reported medium loads lifted } \\
(11-20 \mathrm{~kg}) \text { per day }\end{array}$} & \multicolumn{6}{|c|}{ Frequency of self-reported heavy loads lifted (>20 kg) per day } \\
\hline & \multicolumn{2}{|c|}{0} & \multicolumn{2}{|c|}{$1-10$} & \multicolumn{2}{|c|}{$>10$} \\
\hline & $O R^{a}$ & $95 \% \mathrm{Cl}$ & $O R^{a}$ & $95 \% \mathrm{Cl}$ & $O R^{a}$ & $95 \% \mathrm{Cl}$ \\
\hline 0 & 1.00 & reference & 1.16 & $0.95-1.42$ & 1.64 & $1.06-2.54$ \\
\hline $1-10$ & 1.03 & $0.96-1.11$ & 1.31 & $1.21-1.43$ & 1.55 & $1.01-2.37$ \\
\hline$>10$ & 1.24 & $1.05-1.45$ & 1.41 & $1.15-1.73$ & 1.55 & $1.30-1.85$ \\
\hline
\end{tabular}

${ }^{a}$ Adjusted for maternal age at conception, parity, occupational status, pre-pregnancy body mass index, smoking during pregnancy, leisure time physical activity during pregnancy, leisure time heavy lifting, and working hours. Test for interaction $(P=0.7185)$

supposed to take place around the $12-16^{\text {the }}$ week of the pregnancy (20). At that time, information on exposure and outcome may not have been temporally separated if some women already suffered from pelvic pain when they answered the questions on occupational lifting. Hence, if the reported exposure information depended on disease occurrence, recall bias might have inflated the effect estimates. Alternatively, the actual exposure to occupational lifting could have been reduced due to pain in the present or a previous pregnancy.

In a sub-analysis, we stratified by the timing of the interview. We found a higher risk among women interviewed in the second and the third compared to first trimester. These findings led to some concerns. First, if a woman had already been referred to other work tasks due to pelvic pain before the time of the interview and reported no lifting during the interview, this would tend to weaken the association. Second, due to recall bias some women might have over-reported their actual lifting. Finally, if there is a true association between occupational lifting in the third trimester, this could be the late gestational timing per se or, perhaps more likely, lifting in the last term of the pregnancy may represent an exposure to lifting throughout pregnancy. These mechanisms may influence the association in different directions. In this respect, we did a sub-analysis where no important changes of risk estimates were observed when we restricted the analysis to women who did not report any present or previous muscle or joint diseases. Hence, these findings may indicate no recall bias. However, the results should be interpreted with caution, and the problems regarding onset of pelvic pain should be considered carefully when designing future studies.

Differential item response may be another source of misclassification of occupational lifting. Women in certain types of occupations may perceive lifting the same amount of loads and the same frequency of lifts differently from women in other occupations (23). In order to address differential item response, we used independent exposure estimates from Rubak's JEM (17). When we 
Table 4. Joint effects of physical activity and self-reported occupational lifting on risk of pelvic pain during pregnancy. The Danish National Birth Cohort, 1996-2002. [OR=0dds ratio: $95 \% \mathrm{Cl}=95 \%$ confidence interval]

\begin{tabular}{|c|c|c|c|c|c|c|}
\hline & \multicolumn{6}{|c|}{ Leisure time physical activity (minutes/week) } \\
\hline & \multicolumn{2}{|c|}{0} & \multicolumn{2}{|c|}{$1-60$} & \multicolumn{2}{|c|}{$>60$} \\
\hline & $\mathrm{OR}^{\mathrm{a}}$ & $95 \% \mathrm{Cl}$ & $O R^{a}$ & $95 \% \mathrm{Cl}$ & $\mathrm{OR}^{\mathrm{a}}$ & $95 \% \mathrm{Cl}$ \\
\hline \multicolumn{7}{|c|}{ Any self-reported lifting b } \\
\hline No & 1.00 & reference & 0.85 & $0.78-0.92$ & 0.76 & $0.70-0.81$ \\
\hline Yes & 1.10 & $1.03-1.18$ & 1.17 & $1.03-1.32$ & 1.02 & $0.92-1.13$ \\
\hline \multicolumn{7}{|c|}{ Self-reported total load lifted (kg/day) } \\
\hline 0 & 1.00 & reference & 0.85 & $0.78-0.92$ & 0.75 & $0.70-0.81$ \\
\hline $15-100$ & 1.00 & $0.92-1.09$ & 1.01 & $0.85-1.20$ & 0.88 & $0.76-1.01$ \\
\hline $101-200$ & 1.10 & $0.97-1.24$ & 1.25 & $0.96-1.65$ & 1.12 & $0.91-1.38$ \\
\hline $201-500$ & 1.30 & $1.15-1.47$ & 1.55 & $1.18-2.03$ & 1.39 & $1.12-1.72$ \\
\hline $501-1000$ & 1.40 & $1.15-1.70$ & 1.58 & $0.98-2.55$ & 1.08 & $0.72-1.61$ \\
\hline$>1000$ & 1.33 & $1.00-1.77$ & 1.00 & $0.48-2.09$ & 0.95 & $0.46-1.97$ \\
\hline
\end{tabular}

${ }^{a}$ Adjusted for maternal age at conception, parity, social status, pre-pregnancy body mass index, smoking during pregnancy, leisure time heavy lifting, and working hours.

${ }^{\mathrm{b}}$ Test for interaction any occupational lifting $(\mathrm{P}=0.0009)$

Table 5. Adjusted odds ratios (OR) for pelvic pain during pregnancy according to self-reported occupational lifting stratified according to time of completion of the pregnancy interview, the Danish National Birth Cohort, 1996-2002. [OR=odds ratio; 95\% Cl=95\% confidence interval]

\begin{tabular}{|c|c|c|c|c|c|c|}
\hline & \multicolumn{6}{|c|}{ Trimester at the time of pregnancy interview a } \\
\hline & \multicolumn{2}{|c|}{$1^{\text {st }}$} & \multicolumn{2}{|c|}{$2^{\text {nd }}$} & \multicolumn{2}{|c|}{$3^{\text {rd }}$} \\
\hline & $O R^{a}$ & $95 \% \mathrm{Cl}$ & $\mathrm{OR}{ }^{\mathrm{a}}$ & $95 \% \mathrm{Cl}$ & $O R^{a}$ & $95 \% \mathrm{Cl}$ \\
\hline \multicolumn{7}{|c|}{ Any self-reported lifting b } \\
\hline No & 1.00 & reference & 1.00 & reference & 1.00 & reference \\
\hline Yes & 1.05 & $0.89-1.24$ & 1.18 & $1.12-1.26$ & 2.33 & $1.48-3.65$ \\
\hline
\end{tabular}

${ }^{a}$ Adjusted for maternal age at conception, parity, social status, pre-pregnancy body mass index, smoking during pregnancy, physical activity during pregnancy, leisure time heavy lifting, and working hours.

${ }^{\mathrm{b}}$ Test for interaction $(\mathrm{P}=0.1047)$.

used the JEM, we found no association between occupational lifting and pelvic pain during pregnancy. The JEM was developed for a population-based study on hip and knee osteoarthritis, and for that purpose it was especially relevant to identify heavily exposed jobs. In the JEM, $>50 \%$ of all occupational titles were assessed as being minimally exposed. To the extent that these groups were actually exposed to lifting, the exposure-response relations based on the JEM would underestimate the true associations. For jobs that include lifting patients, the total load lifted per day may have been relatively difficult to assess, which may imply exposure misclassification in the JEM especially for women. Moreover, the JEM did not provide different estimates for men and women within the same occupation. Women in highly exposed jobs may actually perform tasks other than those performed by their male colleagues and, thus, be less exposed; again this would lead to an underestimation of the association. Finally, the JEM could not account for job modifications to minimize the physical exposures during pregnancy. Therefore, we believe, that the self-reported occupational lifting in this case provides a more valid measure than the JEM of the actual loads lifted

Due to the lack of a "golden standard disease definition", it is difficult to measure pelvic pain. In this study, pelvic pain during pregnancy was measured by a screening question six months post partum, and the prevalence of the disease was comparable to the findings in other studies using case definitions based on self-report. Mild degrees of pain should, however, not be captured by our case definition that required pain to interfere with walking, and all cases would probably not fulfill this clinical case criteria. In a nested case-control study on physically demanding working conditions and risk of pelvic pain during pregnancy within the DNBC, three quarters of the women were verified as suffering from the disease. This verification was based on detailed questions on disease symptoms, including pain localization and pain severity when performing different daily activities (2). However, due to difficulties in remembering pain six months back in time, pelvic pain during pregnancy may be either over- or under-reported, but other options for outcome assessment were not available in the present study.

Based on our results, approximately $4 \%$ of all cases of pelvic pain during pregnancy could be prevented if all 
pregnant women were protected from any occupational lifting. Thus, in terms of public health, it can be argued that only a small proportion of pain can be prevented by eliminating occupational lifting during pregnancy. Nevertheless, the contribution of this study is to indicate that some pelvic pain during pregnancy may be preventable.

In conclusion, these results suggest that occupational lifting during pregnancy is associated with an increased risk of pelvic pain. An exposure-response relation was observed for self-reported total load lifted, and the risk increased as the loads lifted and the frequency of lifts increased.

\section{Acknowledgments}

The Danish National Research Foundation has established the Danish Epidemiology Science Centre that initiated and created the DNBC. The cohort is furthermore a result of a major grant from this foundation. Additional support for the DNBC is obtained from the Pharmacy Foundation, the Egmont Foundation, the March of Dimes Birth Defects Foundation, and the Augustinus Foundation. This study is supported by a grant from the Danish Working Environment Research Foundation (contract 20080016458) to the MINERVA project. The authors thank Tine Steen Rubak for permission to use the JEM developed for the study on physical workload and risk of hip replacement.

The authors declare no conflict of interest.

\section{References}

1. Figa-Talamanca I. Occupational risk factors and reproductive health of women. Occup Med (Lond). 2006;56(8):521-31. http://dx.doi.org/10.1093/occmed/kq1114.

2. Juhl M, Andersen PK, Olsen J, Andersen AM. Psychosocial and physical work environment, and risk of pelvic pain during pregnancy. A study within the Danish national birth cohort. J Epidemiol Community Health. 2005;59(7):580-5. http:// dx.doi.org/10.1136/jech.2004.029520

3. Wergeland E, Strand K. Working conditions and prevalence of pre-eclampsia, Norway 1989. Int J Gynaecol Obstet. 1997;58(2):189-96. http://dx.doi.org/10.1016/S00207292(97)00083-0.

4. Nurminen T, Lusa S, Ilmarinen J, Kurppa K. Physical work load, fetal development and course of pregnancy. Scand J Work Environ Health. 1989;15(6):404-14. http://dx.doi. org/10.5271/sjweh.1832.

5. Tophoj A. Graviditetogsygefravær [Pregnancy-related and work-related sick leave of pregnant women]. Ugeskr Laeger. 1994;156(43):6359-65.
6. Rasmussen OB, Sorensen AU, Nielsen CV. Sygemeldingafgravidei Fjends Kommune 1987-1990 [Sick leave for pregnant women in the municipality of Fjend 19871990]. Ugeskr Laeger. 1996;158(49):7069-73.

7. Vleeming A, Albert HB, Ostgaard HC, Sturesson B, Stuge B. European guidelines for the diagnosis and treatment of pelvic girdle pain. Eur Spine J. 2008;17(6):794-819. http://dx.doi. org/10.1007/s00586-008-0602-4.

8. Hansen A, Jensen DV, Wormslev M, Minck H, Johansen S, Larsen EC, et al. Symptom-giving pelvic girdle relaxation during pregnancy. II: Symptoms and clinical signs. Acta Obstet Gynecol Scand. 1999;78(2):111-5. http://dx.doi.org/10.1080/ j.1600-0412.1999.780207.x.

9. Mogren IM, Pohjanen AI. Low back pain and pelvic pain during pregnancy: prevalence and risk factors. Spine (Phila Pa 1976). 2005;30(8):983-91.

10. Robinson HS, Mengshoel AM, Bjelland EK, Vollestad NK. Pelvic girdle pain, clinical tests and disability in late pregnancy. Man Ther. 2010;15(3):280-5. http://dx.doi. org/10.1016/j.math.2010.01.006.

11. Wu W, Meijer OG, Jutte PC, Uegaki K, Lamoth CJ, Sander de WG, et al. Gait in patients with pregnancy-related pain in the pelvis: an emphasis on the coordination of transverse pelvic and thoracic rotations. Clin Biomech (Bristol, Avon). 2002;17(9-10):678-86.

12. Larsen EC, Wilken-Jensen C, Han-sen A, Jensen DV, Johansen $\mathrm{S}$, Minck $\mathrm{H}$, et al. Symptom-giving pelvic girdle relaxation during pregnancy. I: Prevalence and risk factors. Acta Obstet Gynecol Scand. 1999;78(2):105-10. http://dx.doi. org/10.1080/j.1600-0412.1999.780206.x.

13. Endresen EH. Pelvic pain and low back pain in pregnant women-an epidemiological study. Scand J Rheumatol. 1995;24(3):13541. http://dx.doi.org/10.3109/03009749509099301.

14. Ostgaard HC, Andersson GB, Schultz AB, Miller JA. Influence of some biomechanical factors on low-back pain during pregnancy. Spine (Phila Pa 1976). 1993;18(1):61-5.

15. Mogren IM. Previous physical activity decreases the risk of low back pain and pelvic pain during pregnancy. Scand J Public Health. 2005;33(4):300-6.

16. Olsen J, Melbye M, Olsen SF, Sorensen TI, Aaby P, Andersen AM, et al. The Danish National Birth Cohort--its background, structure and aim. Scand J Public Health. 2001;29(4):300-7. http://dx.doi.org/10.1177/14034948010290040201.

17. Rubak T. Cumulative physical exposures in the work environment as a risk factor for primary osteoarthiritis leading to total hip placement Faculty of Health Sciences, Aarhus University; 2010.

18. Albert HB, Godskesen M, Korsholm L, Westergaard JG. Risk factors in developing pregnancy-related pelvic girdle pain. Acta Obstet Gynecol Scand. 2006;85(5):539-44. http://dx.doi. org/10.1080/00016340600578415.

19. van de Pol G, Van Brummen HJ, Bruinse HW, Heintz AP, Van Der Vaart CH. Pregnancy-related pelvic girdle pain in the Netherlands. Acta Obstet Gynecol Scand. 2007;86(4):416-22. http://dx.doi.org/10.1080/00016340601151683. 
20. Wu WH, Meijer OG, Uegaki K, Mens JM, van Dieen JH, Wuisman PI, et al. Pregnancy-related pelvic girdle pain (PPP), I: Terminology, clinical presentation, and prevalence. Eur Spine J. 2004;13(7):575-89. http://dx.doi.org/10.1007/ s00586-003-0615-y.

21. Kirkwood R, Sterne J. Essential medical statistics. 2nd ed. Blacweel Science Ldt; 2003.

22. Vanderweele TJ, Mumford SL, Schisterman EF.
Conditioning on intermediates in perinatal epidemiology. Epidemiology. 2012;23(1):1-9. http://dx.doi.org/10.1097/ EDE.0b013e31823aca5d.

23. Armstrong BG. Effect of measurement error on epidemiological studies of environmental and occupational exposures. Occup Environ Med. 1998;55(10):651-6. http://dx.doi.org/10.1136/ oem.55.10.651.

Received for publication: 6 February 2012 